\title{
Distinct activation thresholds of human conventional and innate-like memory T cells
}

\author{
Chloe K. Slichter, ${ }^{1,2}$ Andrew McDavid, ${ }^{1,3}$ Hannah W. Miller, ${ }^{1}$ Greg Finak, ${ }^{1}$ Brenda J. Seymour, ${ }^{1}$ \\ John P. McNevin, ${ }^{1}$ Gabriela Diaz, ${ }^{1}$ Julie L. Czartoski, ${ }^{1}$ M. Juliana McElrath,, ${ }^{1,2,5,6}$ Raphael Gottardo, ${ }^{1,2,4}$ \\ and Martin Prlic ${ }^{1,2}$ \\ 'Vaccine and Infectious Disease Division, Fred Hutchinson Cancer Research Center, Seattle, Washington, USA. \\ 2Department of Global Health and ${ }^{3}$ Department of Statistics, University of Washington, Seattle, Washington, USA. \\ ${ }^{4}$ Department of Medicine and ${ }^{5}$ Department of Laboratory Medicine, University of Washington, Seattle, Washington, USA. \\ ${ }^{6}$ Public Health Sciences Division, Fred Hutchinson Cancer Research Center, Seattle, Washington, USA.
}

\begin{abstract}
Conventional memory CD8 ${ }^{+} \mathrm{T}$ cells and mucosal-associated invariant T cells (MAIT cells) are found in blood, liver, and mucosal tissues and have similar effector potential following activation, specifically expression of IFN- $\gamma$ and granzyme B. To better understand each subset's unique contributions to immunity and pathology, we interrogated inflammation- and TCR-driven activation requirements using human memory $\mathrm{CD8}^{+} \mathrm{T}$ and MAIT cells isolated from blood and mucosal tissue biopsies in ex vivo functional assays and single cell gene expression experiments. We found that MAIT cells had a robust IFN- $\gamma$ and granzyme B response to inflammatory signals but limited responsiveness when stimulated directly via their TCR. Importantly, this is not due to an overall hyporesponsiveness to TCR signals. When delivered together, TCR and inflammatory signals synergize to elicit potent effector function in MAIT cells. This unique control of effector function allows MAIT cells to respond to the same TCR signal in a dichotomous and situation-specific manner. We propose that this could serve to prevent responses to antigen in noninflamed healthy mucosal tissue, while maintaining responsiveness and great sensitivity to inflammation-eliciting infections. We discuss the implications of these findings in context of inflammation-inducing damage to tissues such as BM transplant conditioning or HIV infection.
\end{abstract}

Conflict of interest: The authors have declared that no conflict of interest exists.

Submitted: January 7, 2016 Accepted: April 26, 2016 Published: June 2, 2016

Reference information: JCI Insight. 2016;1(8):e86292. doi:10.1172/jici.nsight.86292.

\section{Introduction}

Three major categories of antigen have been identified so far that can be recognized by different $\mathrm{T}$ cell subsets: (i) conventional T cells recognize peptides in the context of MHC class I or II, (ii) natural killer T cells (NKT cells) recognize lipids/glycolipids in the context of CD1, and (iii) mucosal-associated invariant $\mathrm{T}$ cells (MAIT cells) recognize bacterially derived metabolites in the context of MHC class I-related protein (MR1) (1). The respective roles of TCR signals and proinflammatory cytokines in regulating activation of primary human MAIT cells have not been well characterized to date.

Mouse model studies introduced the concept of inflammation-driven, $\mathrm{T}$ cell receptor-independent (TCR-independent) activation of memory $\mathrm{CD}^{+} \mathrm{T}$ cells (2-5). More recent studies further defined the mechanisms and inflammatory cues leading to this "bystander-activation" of memory $\mathrm{T}$ cells $\left(\mathrm{T}_{\mathrm{mem}}\right)(6,7)$ and demonstrate that human $\mathrm{T}_{\text {mem }}$ also become bystander activated in many inflammation-inducing scenarios, including infection and cancer immunotherapy (8-14). Bystander activation of $\mathrm{T}_{\mathrm{mem}}$ leads to rapid secretion of IFN- $\gamma$ and enhanced early pathogen clearance $(4,5,7,15)$, which suggests that $\mathrm{T}_{\text {mem }}$ contribute to host immunity in a TCR-independent fashion. Bystander-activated $\mathrm{T}_{\text {mem }}$ also express granzyme $\mathrm{B}$ and can kill NKG2D ligand-expressing target cells in an NKG2D-dependent, innate-like manner (7). This mechanism of target cell elimination can help curtail pathogen spread following an infection (7) but can also exacerbate pathology in the context of an infection $(16,17)$ and autoimmunity $(18)$.

These cytokine-driven, innate-like responses by conventional $\mathrm{T}_{\mathrm{mem}}$ are similar to the inflammationdriven activation of NKT cells (19-21) and MAIT cells (22). While NKT cells are fairly rare in human blood and tissue, MAIT cells are quite abundant and make up $1 \%-8 \%$ of T cells in blood and mucosal tis- 
sues and $20 \%-45 \%$ of T cells in the liver $(23,24)$. Importantly, MAIT cells have a memory-like phenotype (23) and effector functions, including cytotoxicity, that are comparable with conventional memory CD8 ${ }^{+} \mathrm{T}$ cells. MAIT cells can be activated by the same inflammatory signals as conventional $\mathrm{T}_{\text {mem }}$ to express IFN- $\gamma$ and granzyme B $(22,25)$.

Changes in MAIT cell abundance or location have been described in a series of studies including chronic infections, cancer, and autoimmune disorders, indicating that MAIT cells respond and are thus relevant in a wide array of conditions $(26,27)$. There is substantial overlap in activation requirements, functional program, and location between conventional memory $\mathrm{CD} 8^{+} \mathrm{T}$ cells and MAIT cells; thus, we sought to stringently define unique activation and functional characteristics to better understand each subset's potentially unique role and contribution to immunity and pathology.

While conventional memory $\mathrm{CD}^{+} \mathrm{T}$ cells and MAIT cells appear to have similar inflammation-driven responses, it is unclear if MAIT cells respond like conventional memory $\mathrm{CD}^{+} \mathrm{T}$ cells when stimulated via their TCR. We hypothesized that the role of TCR-mediated signals for primary human MAIT cell and conventional memory $\mathrm{CD} 8^{+} \mathrm{T}$ cell activation are different due to the following consideration: since MAIT cells recognize bacterial metabolites that are produced by commensal as well as pathogenic bacteria (28, 29), specific activation requirements must exist to allow for dichotomous responses. Moreover, assuming that a conventional memory $\mathrm{CD} 8^{+} \mathrm{T}$ cell has a more focused antigenic specificity than a MAIT cell (specific peptide sequence versus a metabolite), we reasoned that TCR-mediated memory CD8 ${ }^{+} \mathrm{T}$ cell responses are inherently more restricted, and conventional $\mathrm{T}_{\text {mem }}$ may thus have a different TCR-activation threshold compared to MAIT cells.

We report here that, in contrast to conventional memory $\mathrm{CD} 8^{+} \mathrm{T}$ cells, even a strong $\mathrm{TCR}+$ costimulatory signal is not sufficient to induce robust and sustained MAIT cell effector function. Importantly, we demonstrate that inflammatory signals and TCR signals synergize to induce MAIT cell effector function.

To better understand MAIT cell characteristics and contributions to immunity at the site of the mucosa, we used single cell gene expression analysis of MAIT cells isolated from blood and mucosal tissue biopsies. We did not observe direct ex vivo effector function in MAIT cells isolated from healthy mucosal tissues; however, we found that MAIT cells in mucosal tissue have pronounced proinflammatory/activating potential. This suggests that mucosal MAIT cells are poised to respond rapidly and further underlines the need for tight functional control to prevent unwanted effector responses. We propose that the previously unappreciated requirement for inflammatory cues to acquire MAIT cell effector function provides an explanation for the coexistence of MAIT cells and commensals in mucosal tissues without eliciting pathology (TCR signal without inflammation), while maintaining responsiveness to pathogens (TCR signal plus inflammation). Our findings also have important implications for understanding changes in MAIT cell function following BM transplant conditioning (30) or HIV infection (31-34), which damage the gastrointestinal luminal integrity and elicit inflammatory responses.

\section{Results}

TCR signals are not sufficient to directly induce MAIT cell effector function. The functional properties of MAIT cells have typically been examined following stimulation of whole peripheral blood mononuclear cells (PBMC) or enrichment of $\mathrm{CD} 161{ }^{\text {hi }} \mathrm{CD} 8^{+}$cells. We wanted to stringently define the ability of purified MAIT cells and conventional $\mathrm{T}_{\mathrm{mem}}\left(\mathrm{CD}^{+} \mathrm{T}_{\mathrm{mem}}\right)$ to directly respond to cytokine- and TCR-mediated stimuli. We tested 2 different approaches to purify MAIT cells. We first developed a sorting strategy that relies on the CCR6 ${ }^{\text {hi }}$ expression levels of MAIT cells (23) (Figure 1A). Using this strategy, we typically increased MAIT cell purity to $>90 \%$ (Figure $1 \mathrm{~A}$, right panel). To further increase the sort purity, we next included an antibody against V $\alpha 7.2$ (the invariant MAIT TCR $\alpha$ chain) in the sorting panel and further increased the cell purity to greater than $95 \%$ (data not shown). Importantly, comparing these 2 methods, we found no evidence that the anti-V $\alpha 7.2$ antibody significantly altered MAIT cell responses (Supplemental Figure 1; supplemental material available online with this article; doi:10.1172/jci.insight.86292DS1) and we thus continued to use the Va7.2 sorting strategy for greatest MAIT cell purity. Conventional $\mathrm{CD}^{+} \mathrm{T}_{\text {mem }}$ were sorted as $\mathrm{CD}^{+} \mathrm{CD} 45 \mathrm{RO}^{+} \mathrm{V} \alpha 7.2^{-}$cells (see Methods for full sort panel).

Sorted MAIT and conventional $\mathrm{CD}^{+} \mathrm{T}_{\text {mem }}$ were incubated for 6,12 , or 24 hours with a combination of the cytokines IL-12, IL-15, and IL-18 (IL-12/15/18) or with anti-CD3/CD28 beads (TCR). Anti-CD3/ $\mathrm{CD} 28$ beads allowed us to compare the MAIT cell response to the $\mathrm{CD} 8^{+} \mathrm{T}_{\text {mem }}$ population and deliver a well-defined and standardized TCR + costimulatory signal across stimulation conditions and cell types. 
A Pre-sort: Gated on $\mathrm{CD} 8^{+}$cells
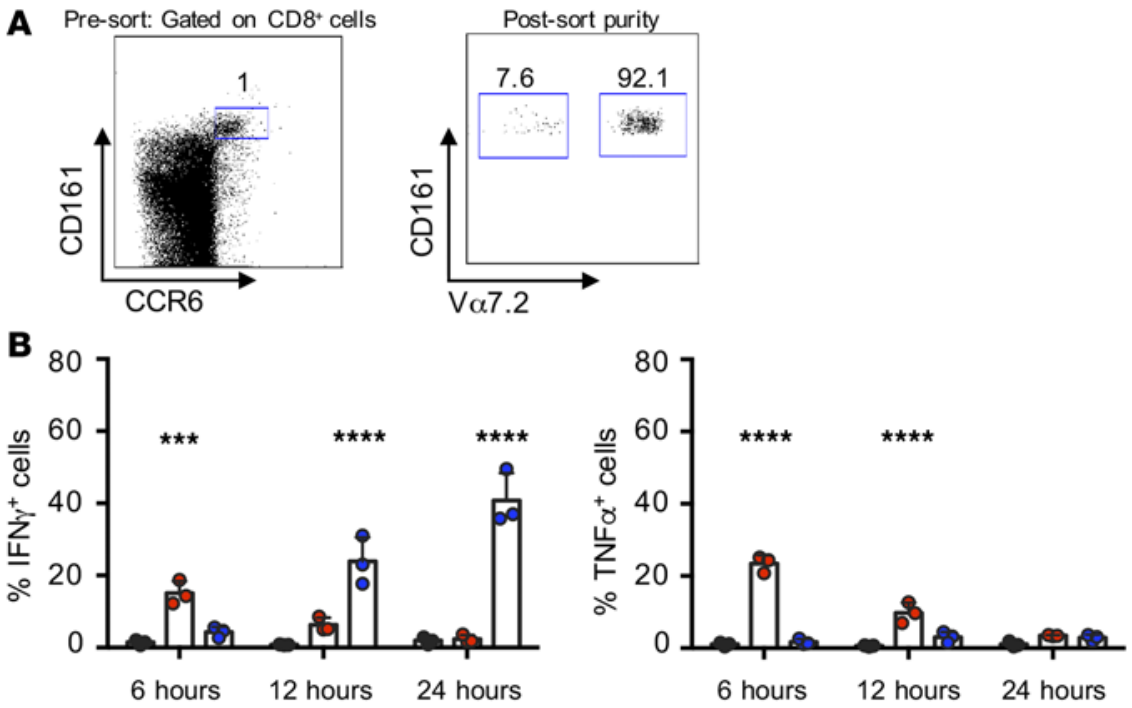

\author{
MAIT $\left(\mathrm{CD} 8^{+} \mathrm{CD} 161^{\text {hi }} \mathrm{V} \alpha 7.2^{+}\right)$

No stimulation (NS)
TCR
IL-12/15/18
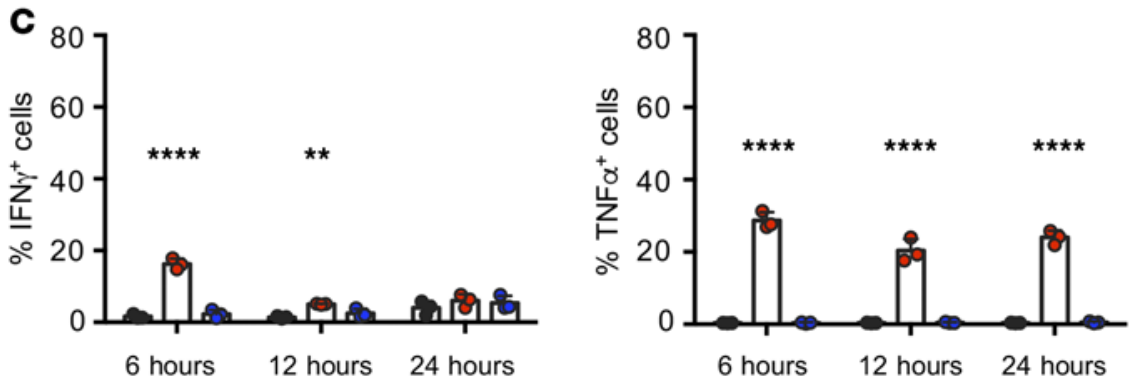

$\mathrm{CD} 8+\mathrm{T}_{\text {mem }}\left(\mathrm{CD}^{+} \mathrm{CD}^{+} 5 \mathrm{RO}^{+}\right)$ No stimulation (NS) OTCR OIL-12/15/18

Figure 1. A TCR signal alone is not sufficient for sustained effector function in MAIT cells. (A) A representative flow plot of the CD161 ${ }^{\text {hi } C C R 6^{\text {hi }}}$ MAIT cell population in peripheral blood mononuclear cells (PBMC) after gating on CD8 ${ }^{+} \mathrm{T}$ cells is shown (left panel). MAIT cell purity was confirmed after sorting CD8 ${ }^{+}$CD161 $1^{\text {hi }}$ CCR6 $6^{\text {hi }}$ cells by determining V $\alpha 7.2$ expression in a small aliquot (right panel). (B) Five thousand sorted CD8 ${ }^{+}$CD161 $1^{\text {hiV }} \alpha 7.2^{+}$MAIT cells were rested (no stimulation, black circles); stimulated with anti-CD3/CD28 beads (TCR, red circles) or $100 \mathrm{ng} / \mathrm{ml}$ of IL-12/15/18 (blue circles) for 6 , 12, or 24 hours; and analyzed for expression of IFN- $\gamma$ and TNF- $\alpha(n=3)$. (C) Five thousand sorted CD8 ${ }^{+} C D 45 R 0^{+} V \alpha 7.2^{-}$memory $T_{\text {cells }}\left(C D 8^{+} T_{\text {mem }}\right.$ ) were rested (no stimulation, black circles); stimulated with anti-CD3/CD28 beads (TCR, red circles) or $100 \mathrm{ng} / \mathrm{ml}$ of IL-12/15/18 (blue circles) for 6,12 , or 24 hours; and analyzed for expression of IFN $-\gamma$ and TNF- $\alpha(n=3)$. Data displayed are the average of 2-3 technical replicates for each donor, with each data point representing a single donor $(n=3$, B and $\mathbf{C}$ ); data are displayed as mean \pm SEM (where applicable). ${ }^{* *} P \leq 0.01,{ }^{* * *} P \leq 0.001$, and ${ }^{* * * *} P \leq 0.0001$. $P$ values were determined by comparing treatment conditions to no-stimulation conditions for each time point. Two-way ANOVA, with Dunnett's test for multiple comparisons (B and $\mathbf{C}$ ).

We first analyzed both $\mathrm{T}$ cell subsets by intracellular cytokine staining. MAIT cells responded robustly and expressed IFN- $\gamma$, but not TNF- $\alpha$, as early as 12 hours and at 24 hours after stimulation with recombinant IL-12/15/18 (Figure 1B). In contrast, after TCR stimulation, we observed a short burst of IFN- $\gamma$ and TNF- $\alpha$ expression by MAIT cells at 6 hours, but this effect was lost by 12 hours, indicating that TCR signaling was not sufficient to maintain expression of IFN- $\gamma$ and TNF- $\alpha$. The population of conventional CD ${ }^{+}$ $\mathrm{T}_{\text {mem }}$ was overall less responsive to IL-12/15/18 compared with the MAIT cell population but responded to TCR signaling and produced TNF- $\alpha$ at all time points, while IFN- $\gamma$ production peaked at 6 hours and was still significantly produced at 12 hours before declining by 24 hours after TCR stimulation (Figure 1C).

We were initially surprised to find only limited IFN- $\gamma$ production by MAIT cells in response to TCR signaling (Figure 1B, left panel) and considered that MAIT cells may be hyporesponsive to a direct TCR signal. Thus, we next screened the culture supernatant for secreted cytokines and chemokines by Luminex analysis (Figure 2, A and B) to define which cytokines are secreted within the first 24 hours of activation. IL-12/15/18-stimulated MAIT and conventional CD8 ${ }^{+} \mathrm{T}_{\text {mem }}$ secreted IFN- $\gamma$ but not TNF- $\alpha$. We only detected IFN- $\gamma$ and TNF- $\alpha$ in the supernatant of TCR-stimulated conventional CD $8^{+} \mathrm{T}_{\text {mem }}$ but not TCRstimulated MAIT cells. This further supports the notion that conventional $\mathrm{CD} 8^{+} \mathrm{T}_{\text {mem }}$, but not MAIT cells, secrete significant amounts of effector cytokines following TCR stimulation.

MAIT cells have been reported to express IL-17 upon activation, particularly after prolonged in vitro culture and stimulation (23); however, we could not detect IL-17 in the supernatant of our ex vivo-stim- 

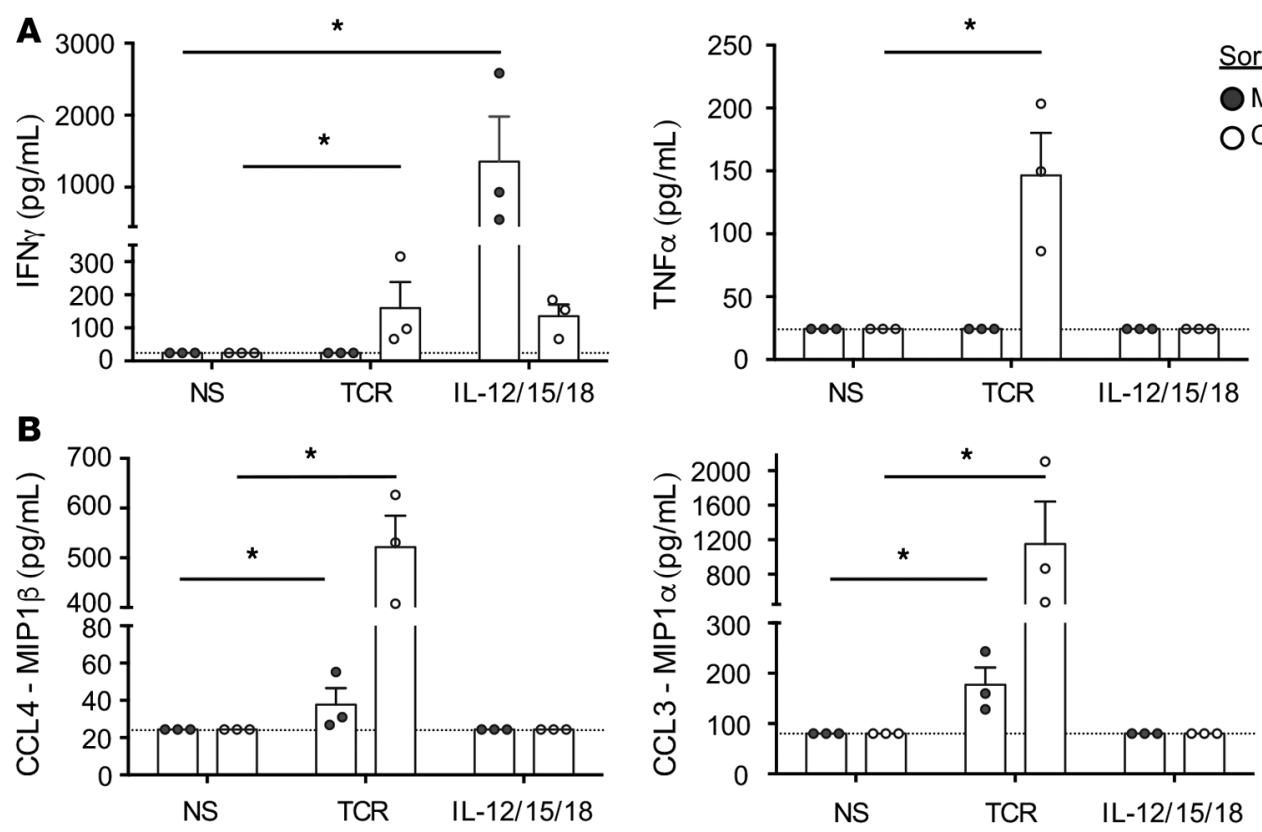

Figure 2. A TCR signal is sufficient to induce effector function in conventional memory CD8+ $\mathbf{T}$ cells but not in MAIT cells. (A and B) Using the supernatant from the same experiments as in Figure 1, MAIT (dark gray circles) or $\mathrm{T}_{\text {mem }}$ (white circles) supernatants were collected after 24 hours of culture and analyzed using Luminex $(n=3)$. Dashed lines denote limit of detection for each analyte. Three different donors were used for each experiment shown. MAIT cell data shown are representative of at least 2 independent, technical replicates. Data are displayed as mean \pm SEM (where applicable). ${ }^{*} P \leq 0.05$. $P$ values were determined by comparing treatment conditions to unstimulated conditions using Mann-Whitney 1-tailed $U$ test (A and B).

ulated MAIT cells, regardless of the experimental stimulation condition (data not shown). Importantly, TCR-mediated signals induced secretion of CCL3 and CCL4 from both MAIT cells and CD8 ${ }^{+} \mathrm{T}_{\text {mem }}$ (Figure 2B), suggesting that MAIT cells are not simply hyporesponsive to TCR stimulation. Finally, we considered the possibility that anti-CD3/CD28 stimulation in the absence of inflammatory stimuli could also lead to induction of immune-suppressive mechanisms as an additional control mechanism. We examined IL-10 secretion but could not detect IL-10 in any of the experimental conditions (data not shown). Together, these data show that inflammation- but not TCR-mediated signals are sufficient to induce MAIT cell effector function. Our approach allowed us to define the signals that act directly on a pure MAIT cell population and compare these results to previously published experiments analyzing MAIT cell responses in the context of other cell types $(22,24)$. When not purified, MAIT cells may be indirectly activated by other (non-MAIT) T cells responding to TCR signals and in turn secrete cytokines that then act on MAIT cells. Since the current model of MAIT cell activation suggests that TCR signals are sufficient for MAIT cell activation based on experiments that examined MAIT cell function in the context of other cell types, we next wanted to explain this seemingly contradictory outcome of our data and these previous studies.

Dissecting direct and indirect MAIT cell activation mechanisms. To distinguish direct from indirect activation mechanisms, we examined how memory CD8 T and MAIT cells respond to inflammatory cues and TCR stimulation in the context of other PBMCs. PBMCs were incubated for 24 hours with a combination of the cytokines IL-12/15/18 or with anti-CD3/CD28 beads (TCR) as described in Figure 1. We found that MAIT cells responded robustly and acquired effector function when PBMC were stimulated with either anti-CD3/CD28 beads or recombinant IL-12/15/18 as previously reported $(22,24)$. CD3/CD28 stimulation of PBMCs induced strong granzyme $\mathrm{B}$ expression in MAIT cells and CD8 $8^{+} \mathrm{T}_{\text {mem }}$ (Figure 3B), but IFN- $\gamma$ expression (Figure 3A) was more limited following CD3/CD28-mediated stimulation compared with cytokine stimulation. We examined MAIT cells again for expression of IL-17 following ex vivo stimulation (with anti-CD3/CD28 beads for 24 hours or PMA/ionomycin for 4 hours; data not shown) but found only minimal to no IL-17 production (data not shown). Together, these data show that MAIT cell activation occurs when bulk PBMCs are activated with anti-CD3/CD28 as previously demonstrated, but - in the context of Figure 1 - this acquisition of effector function is the result of indirect activation and not due to an intrinsic TCR-mediated signal in MAIT cells. Finally, we wanted to define the contribu- 
A

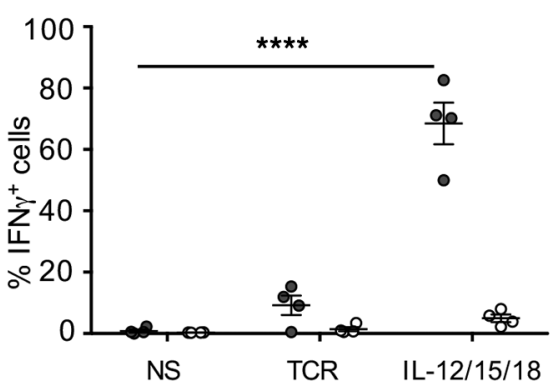

C

Whole PBMC gated on MAIT $\left(\mathrm{CD} 8^{+} \mathrm{CD} 161^{\mathrm{hi}} \mathrm{V} \alpha 7.2^{+}\right)$
B

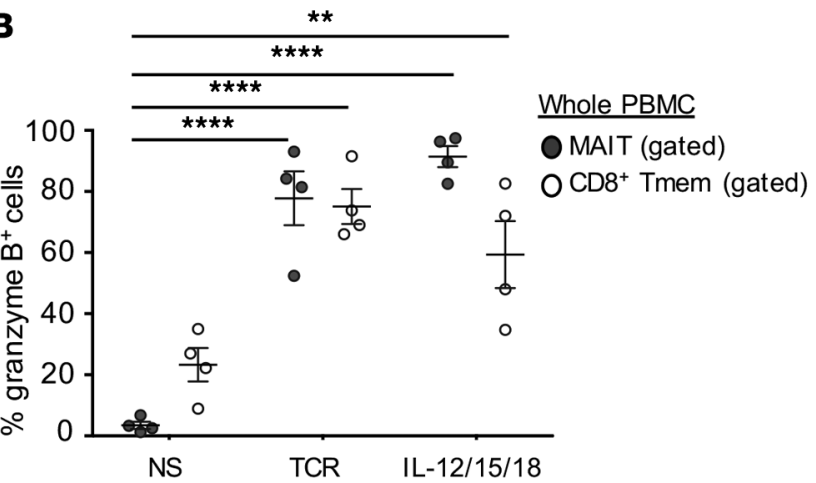

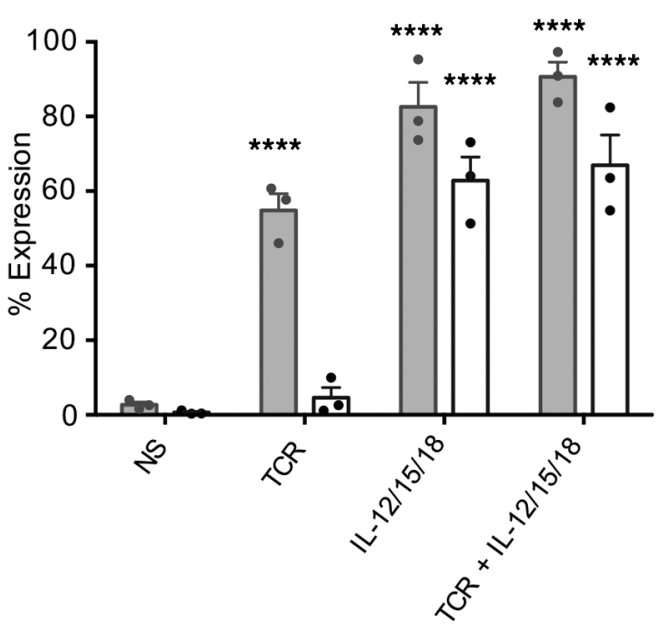

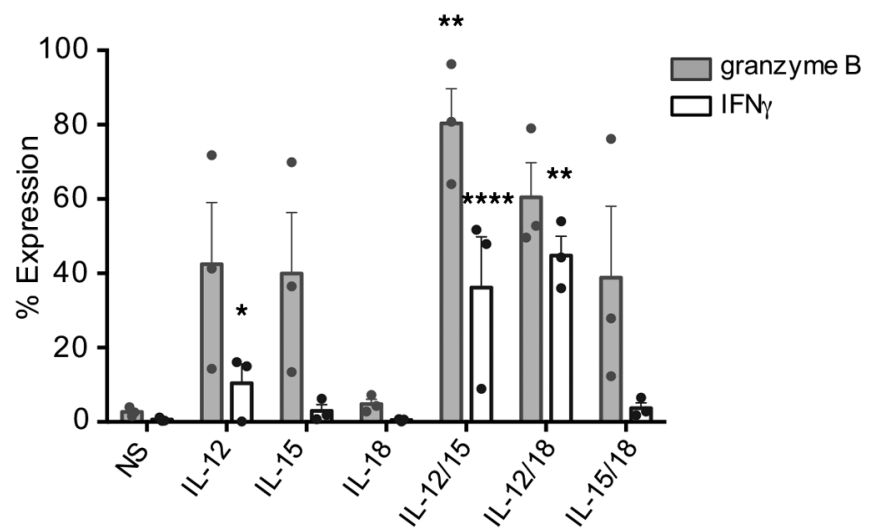

Figure 3. Indirect effects lead to MAIT cell activation when PBMCs are stimulated with anti-CD3/CD28 beads. Whole peripheral blood mononuclear cells (PBMC) stimulated for 24 hours with anti-CD3/CD28 beads (TCR) or $100 \mathrm{ng} / \mathrm{ml} \mathrm{IL-12/15/18} \mathrm{or} \mathrm{left} \mathrm{unstimulated} \mathrm{(NS)} \mathrm{and} \mathrm{analyzed} \mathrm{for} \mathrm{(A)} \mathrm{IFN-} \gamma$ and (B) granzyme B expression by MAIT and CD8 memory T cells (CD8 T cells) $(n=4)$. MAIT cells (dark gray circles) were identified by gating on CD3+CD8 ${ }^{+} C D 161^{\text {hi }} \vee \alpha 7.2^{+}$ cells. $C D 8^{+} T_{\text {mem }}$ (white circles) were identified as $C D 3^{+} C D 8^{+} C D 45 R 0^{+} V \alpha 7.2^{-}$cells. (C) Expression of IFN- $\gamma$ (white bars) and granzyme B (gray bars) by MAIT cells after stimulating PBMC $(n=3)$ for 24 hours with individual cytokines, a combination of cytokines, anti-CD3/CD28 beads (TCR), a combination of cytokines and anti-CD3/CD8 beads, or left unstimulated (NS). Data in $\mathbf{A}$ and $\mathbf{B}$ were generated in separate experiments from data shown in C. Data are displayed as mean \pm SEM (where applicable). ${ }^{* *} P \leq 0.01$ and ${ }^{* * * *} P \leq 0.0001$. $P$ values were determined by comparing treatment conditions to unstimulated conditions using Mann-Whitney $U$ test (A and $\mathbf{B}$ ) or 2-way ANOVA with Dunnett's test for multiple comparisons (C).

tion of each cytokine alone and in combination to the pronounced MAIT cell effector phenotype (Figure 3C). IL-18 was not sufficient to elicit strong effector function, while IL-12 and IL-15 alone could induce granzyme B expression. IL-12 appeared particularly important for IFN- $\gamma$ expression, since IL-12/15 and IL-12/18 stimulation conditions were sufficient for IFN- $\gamma$ production, while IL-15/18 stimulation resulted primarily in granzyme B expression. Interestingly, the combination of IL-12/15/18 resulted in such strong MAIT cell activation that adding TCR signaling had seemingly little impact on further increasing the frequency of responding cells. Together, the experiments so far demonstrate that a TCR signal is not sufficient to directly activate MAIT cells and explain why previous studies missed this characteristic when stimulating bulk PBMCs.

We next sought a better understanding of the role of inflammatory and TCR signals on MAIT cell activation by more closely mimicking physiologically relevant conditions.

Activated monocytes are sufficient to activate MAIT cells in a cell contact-dependent and-independent manner. We set up an experimental system to interrogate the ability of activated professional antigen-presenting cells (APC) to stimulate MAIT cells in a cell contact-dependent versus -independent manner. We used monocytes for this purpose, since monocytes are the most readily available human APC and recent data suggest that monocytes can enter nonlymphoid tissue and recirculate, indicating that they can interact with MAIT cells in blood and tissues (35).

We asked if TLR-stimulated monocytes are sufficient to activate MAIT cells and first wanted to distin- 
A

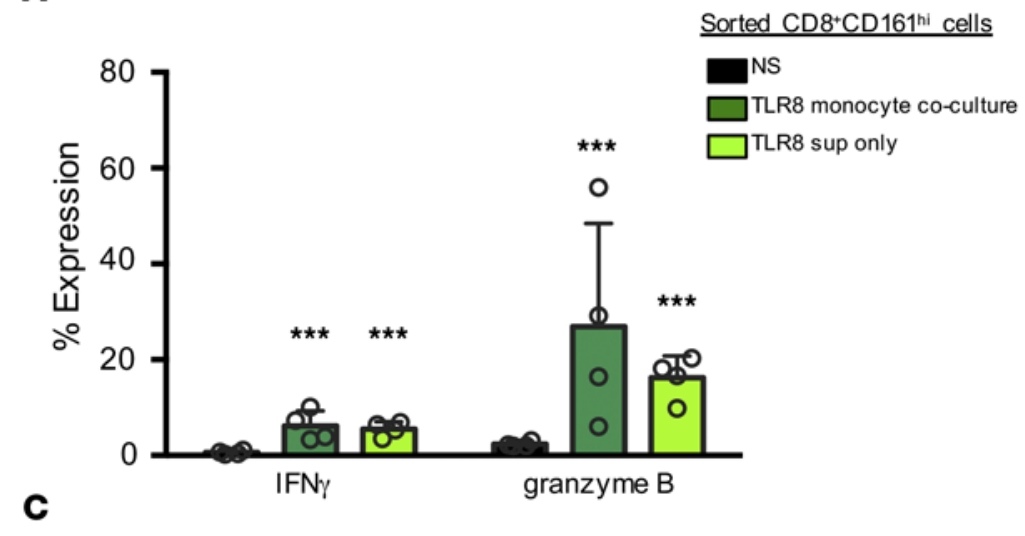

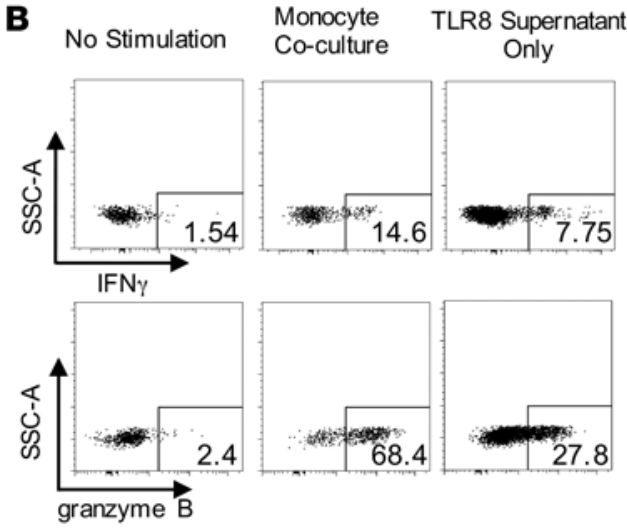
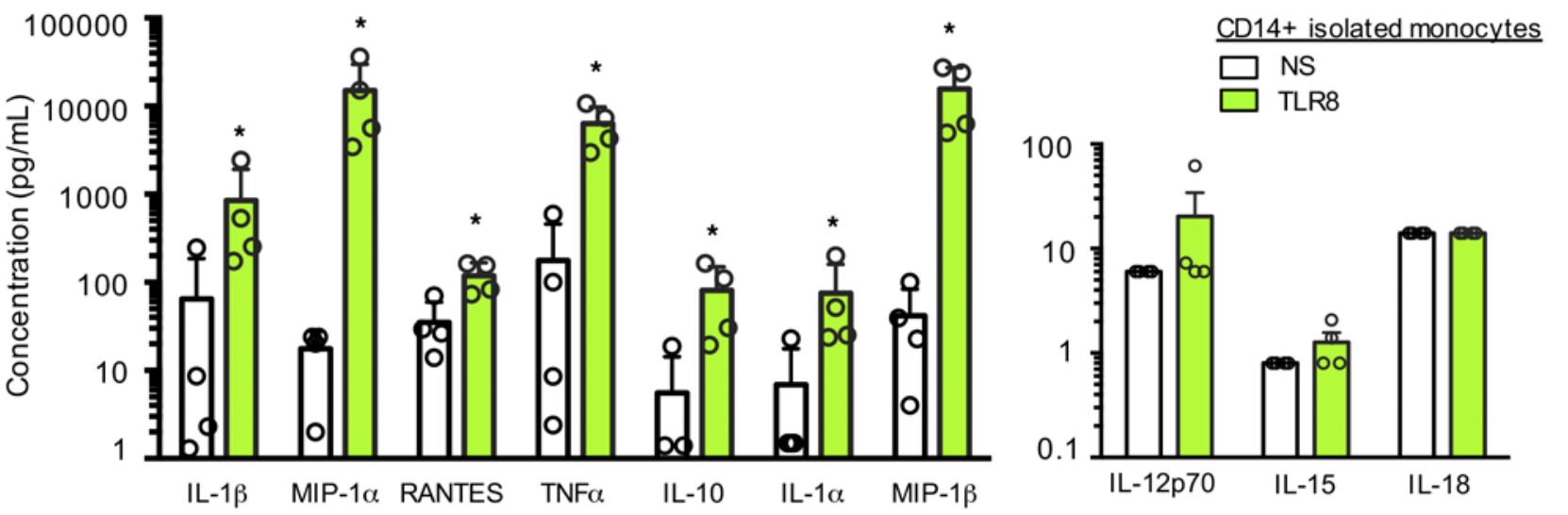

Figure 4. Cell contact-dependent and -independent activation of MAIT cells by TLR-stimulated monocytes. (A) Sorted CD8 ${ }^{+}$CD161 $1^{\text {hi }}$ MAIT cells were cocultured with unstimulated monocytes (NS, black), ssRNA-activated (TLR8 agonist-activated) monocytes (TLR8 monocyte coculture, dark green), or supernatant (sup) from ssRNA-activated monocytes (TLR8 sup only, light green) for 24 hours. After 24 hours of coculture directly with monocytes or with supernatant, MAIT cells (CD8+CD161 hiV $\alpha 7.2^{+}$) were analyzed for IFN- $\gamma$ and granzyme B expression $(n=4)$. (B) A representative FACS plot is shown illustrating IFN- $\gamma$ and granzyme B expression by MAIT cells. (C) Luminex analysis of statistically significant analytes (left panel) or from the cytokines IL-12, IL-15, and IL-18 (right panel) from supernatants collected from CD14+ monocytes that were rested (NS, black) or activated with ssRNA (TLR8, light green) reveal differences in cytokine expression dependent on stimulation $(n=4)$. A lack of a visible error bar in $\mathbf{C}$ (right panel) is due to identical data points. Data shown are displayed as mean \pm SEM (where applicable). ${ }^{*} P \leq 0.05$ and ${ }^{* *} P \leq 0.001$. $P$ values were determined by comparing treatment conditions to unstimulated conditions using Mann-Whitney 1-tailed or 2-tailed $U$ test (A-C).

guish if this happened in a cell contact-dependent or -independent manner. We isolated primary human $\mathrm{CD}_{14}{ }^{+}$monocytes from PBMC and treated the monocytes with a TLR8 agonist (single stranded RNA; ssRNA). After 24 hours, we cocultured them with $C D 8^{+} \mathrm{CD} 161^{\text {hi }}$-sorted MAIT cells from the same donor for an additional 24 hours and then measured cytokine expression by MAIT cells. MAIT cells that were cocultured with unstimulated monocytes for 24 hours served as the negative control. MAIT cells expressed granzyme B and low levels of IFN- $\gamma$ when cocultured with TLR8-activated monocytes (Figure 4A), in line with previous studies $(22,24)$. To determine if cytokines secreted by monocytes are sufficient for activating MAIT cells or if MAIT cell activation is cell contact dependent, we treated monocytes with a TLR8 agonist for 24 hours, removed the supernatant, and cultured MAIT cells in the monocyte culture supernatant for an additional 24 hours. We found that stimulation with supernatant from TLR-activated monocytes led to minimal but detectable granzyme B and IFN- $\gamma$ production by MAIT cells, suggesting that cytokine secretion alone, though not optimal, is sufficient for activation (Figure 4, A and B).

To understand which inflammatory cytokines were secreted by these monocytes, we analyzed the cytokines in the culture supernatant. We determined that IL- $1 \alpha$ and $-\beta$ (Figure 4C) and (in some donors) IL-12 were present, but neither IL-18 nor IL-15 could be detected consistently above background levels (Figure 4C, right panel). These data suggest that the granzyme expression of MAIT cells could be primarily driven by IL-12, as well as by cytokines other than IL-12/15/18.

Together, these data show that an activated APC is sufficient to induce MAIT cell effector function in 
A

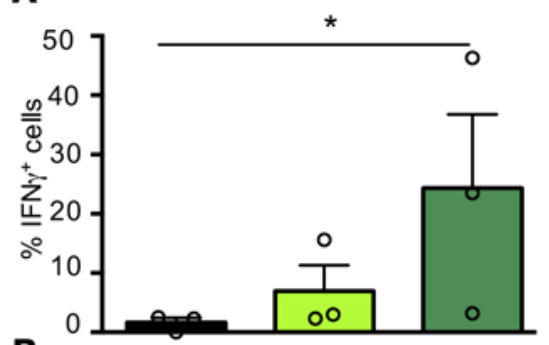

B

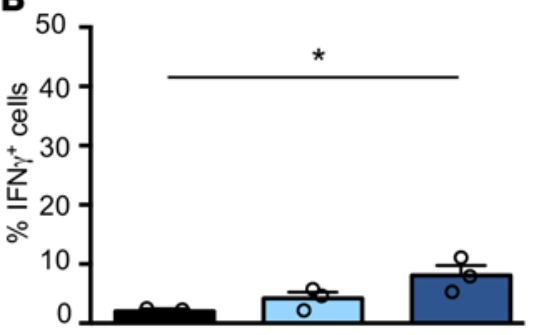

C

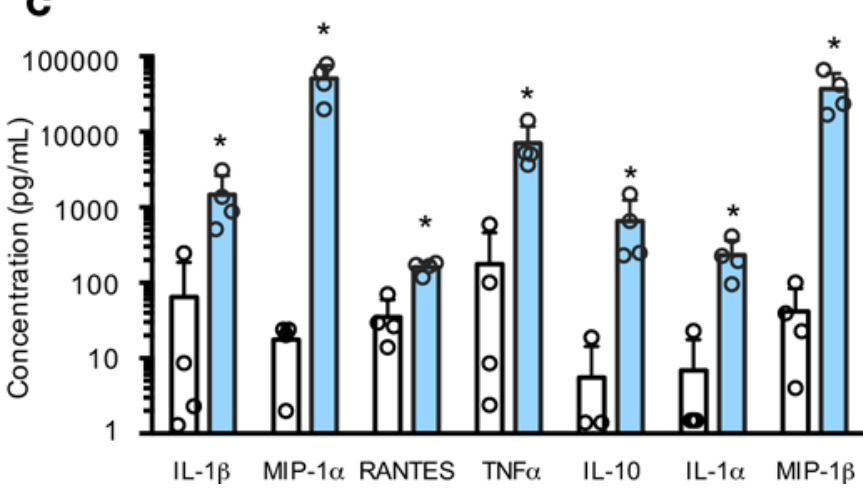

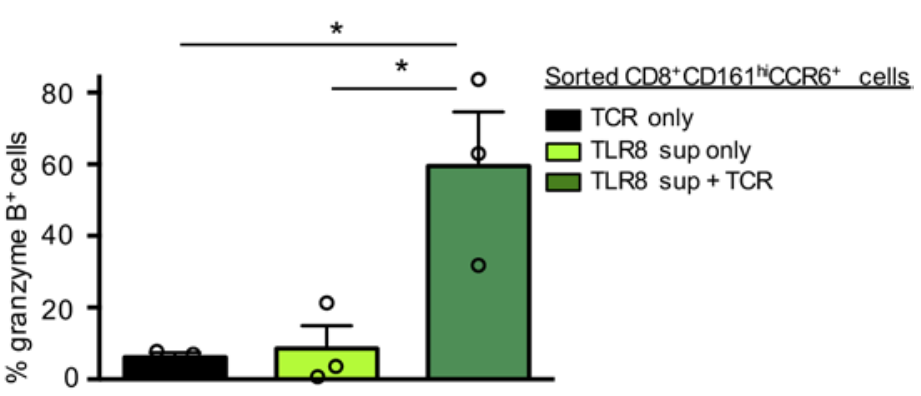

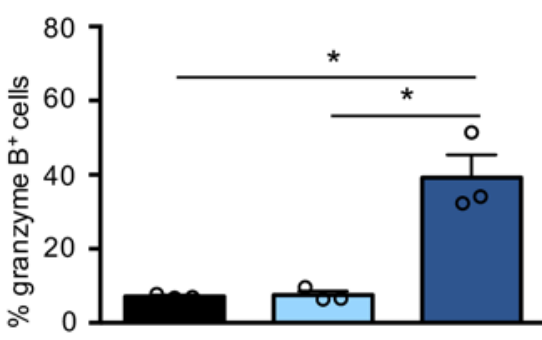

Sorted $\mathrm{CD} 8^{+} \mathrm{CD} 161^{\text {hi }} \mathrm{CCR}^{+}$cells TCR only $\square$ TLR4 sup only $\square$ TLR4 sup + TCR

Figure 5. Inflammatory cytokines and TCR-mediated signals synergize to induce MAIT cell effector function. (A) Monocytes were stimulated with ssRNA (a TLR8 agonist); after 24 hours, supernatant was removed and CD8+CD161 ${ }^{\text {hicCR6 }}$ ( MAIT cells were cocultured with TLR8 supernatant in the presence (TLR8 sup + TCR, dark green) or absence (TLR8 sup only, light green) of anti-CD3/CD28 beads for 24 hours followed by analysis of IFN- $\gamma$ and granzyme B expression $(n=3)$. MAIT cells stimulated only with anti-CD3/CD28 (TCR only) beads for 24 hours are shown in black. (B) Using the same donors and experimental setup as in A, monocytes were stimulated with LPS (a TLR4 agonist) in the presence (TLR4 sup + TCR, dark blue) or absence (TLR4 sup only, light blue) of anti-CD3/CD28 beads for 24 hours followed by analysis of IFN- $\gamma$ and granzyme B expression ( $n=3$ ). MAIT cells stimulated only with anti-CD3/ CD28 (TCR only) beads for 24 hours are shown in black. (C) Luminex analysis of statistically significant analytes (left panel) or from the cytokines IL-12, IL-15, and IL-18 (right panel) from supernatant collected from CD14+ monocytes that were rested (white bars) or activated with LPS (TLR4, light blue bars) reveal differences in cytokine expression dependent on stimulation $(n=4)$. A lack of a visible error bar in $\mathbf{C}$ (right panel) is due to identical data points. Data are displayed as mean \pm SEM (where applicable). ${ }^{*} P \leq 0.05$. $P$ values were determined by comparing treatment conditions to unstimulated or TCR-only conditions using Mann-Whitney 1-tailed or 2-tailed $U$ test $(\mathbf{A}-\mathbf{C})$.

a cell contact-independent manner. Since activation occurred in a cell contact-independent manner, this also suggests that it occurred in the absence of a TCR signal. While a TCR signal had minimal impact on enhancing effector function in IL-12/15/18-stimulated MAIT cells (Figure 3C), we next wanted to define the role of the TCR signal in the context of activated APCs secreting inflammatory cytokines.

Synergy of TCR signaling and inflammatory cytokines. To define how MAIT cells respond to TCR signals in the presence of proinflammatory signals provided by activated APCs, we used a similar experimental setup as in Figure 4A. We isolated primary human $\mathrm{CD} 14^{+}$monocytes from PBMC and treated the monocytes with a TLR8 (ssRNA) or TLR4 (LPS) agonist. Supernatant from unstimulated monocytes was used as a negative control. After 24 hours, we removed the supernatant and added it to $\mathrm{CD} 8^{+} \mathrm{CD} 161{ }^{\text {hi }} \mathrm{CCR} 6{ }^{+}$-sorted MAIT cells from the same donor and examined MAIT cell effector function 24 hours later. There were 3 experimental groups: (i) MAIT cells + TCR signals + negative control supernatant, (ii) MAIT cells + TLRstimulated supernatant, and (iii) MAIT cells + TCR signals + TLR-stimulated supernatant.

We observed minimal expression of granzyme B and IFN- $\gamma$ by MAIT cells when stimulated via their TCR in the presence of supernatant from unstimulated monocytes (Figure 5, A and B). Addition of super- 
Table 1. Single cell gene expression analysis of MAIT cells isolated from blood and mucosal tissue.

\begin{tabular}{|c|c|c|c|c|c|}
\hline & TISSUE & $\mathrm{CD8}^{+} \mathrm{T}$ cell & CD8- MAIT & $\mathrm{CDB}^{+} \mathrm{MAIT}$ & NK cell \\
\hline \multirow[t]{2}{*}{ Donor 1} & Blood & 48 & 47 & 48 & 48 \\
\hline & Mucosal & 43 & 53 & 66 & 0 \\
\hline \multirow[t]{2}{*}{ Donor 2} & Blood & 44 & 47 & 44 & 45 \\
\hline & Mucosal & 46 & 57 & 65 & 0 \\
\hline
\end{tabular}

The number of single cells in each population that passed quality control and were used for subsequent analysis is shown.

natant from TLR4- or TLR8-stimulated monocytes was sufficient to activate MAIT cells and induce detectable amounts of IFN- $\gamma$ and granzyme B (Figure 5, A and B). When both signals were combined (supernatant + TCR signal) the effector response was significantly increased. Given the size of the effector response when both signals are combined, it indicates that TCR signals and inflammatory signals synergize to elicit a robust effector response. This is particularly apparent for granzyme B expression, regardless of the TLR stimulus, and for IFN- $\gamma$ when MAIT cells receive TLR8 supernatant and a TCR signal. To understand how the TLR4 supernatant differs from the TLR8 supernatant, we determined the cytokine profile using the same approach as described in Figure 4C. Overall, many cytokines were secreted at comparable levels (Figure 4C and Figure 5C), including IL-1 $\alpha$ and $-\beta$. Neither IL-18 nor IL-15 could be detected above background levels (Figure $4 \mathrm{C}$ and Figure $5 \mathrm{C}$, right panels) and, in contrast to TLR8 supernatant, we could not detect IL-12 in supernatant from TLR4-stimulated monocytes. These data show that the inflammatory environment controls the extent of effector function, but regardless of the specific inflammatory milieu, there is strong evidence for synergy between inflammatory signals and TCR signals in inducing MAIT cell effector function.

Thus far, all experiments were done with MAIT cells isolated from PBMCs. We considered that MAIT cells located in mucosal tissues may have decreased functional potential that could serve as a cell intrinsic mechanism to avoid unwanted effector responses within tissues. Thus, we wanted to determine functional commonalities and potential differences of MAIT cell populations in blood and mucosal tissue.

MAIT cells in the mucosal tissue are poised to respond more rapidly than their blood counterparts. We considered the possibility that a decrease in MAIT cell effector potential, concomitant with mucosal tissue residency, could serve as a potential additional or alternative safety mechanism to curtail unwanted effector responses against commensal antigen. Due to the limited availability of mucosal tissue from healthy donors restricting the ability to perform direct ex vivo stimulation assays, we chose a single cell gene expression analysis approach to answer this question as comprehensively as possible. Although CD $8 \alpha^{+}$MAIT cells are typically the predominant population, MAIT cells are also found in the CD4-CD8- (double negative; DN) $\mathrm{CD}^{+}$ population (36). Aside from this phenotypic difference, the functional differences of MAIT cell populations are not well understood, and it is furthermore unclear whether populations with distinct functional properties exist in mucosal tissues. We compared gene expression profiles from $\mathrm{CD}^{+}$and $\mathrm{CD} 8^{-} \mathrm{MAIT}$ cells, conventional $\mathrm{CD}^{+} \mathrm{T}$ cells (all CD8 T cells, i.e., naive and memory), and NK cells isolated from peripheral blood and mucosal tissue (CD8 ${ }^{+}$MAIT, CD8 $8^{-}$MAIT and CD $8^{+} \mathrm{T}$ cells only) of 2 healthy donors (Table 1). For these experiments, anti-CD3 and anti-Va7.2 antibodies were included in the sorting panel to ensure optimal sort purity. Expression of 145 genes in each single cell was determined using the nCounter NanoString platform. The NanoString technology relies on a capture probe and a barcoded reporter probe to detect gene transcripts of interest. Using a 2-probe detection system ensures specificity, and transcript abundance is measured by single molecule imaging of the reporter probe. We specifically interrogated the expression pattern of 119 genes controlling or indicating inflammation, activation, and migration (IAM) status of a cell, as well as a set of 26 genes for quality control. CD8 $8^{+} \mathrm{T}$ and NK cells served as reference populations and were also included to facilitate interpretation of the data. Both MAIT cell populations had a distinct gene expression signature compared with conventional $\mathrm{CD} 8^{+} \mathrm{T}$ and NK cells in the blood, indicating their distinct functional properties (Figure 6A). We next compared the 2 MAIT cell populations from each tissue to each other to determine their relationship. We found that the 2 MAIT cell populations share extensive transcriptional overlap in mucosal tissue (Figure 6B, $y$-axis discriminant) and blood (Figure 6C, $y$-axis discriminant), but differences in the transcriptional profile became apparent when comparing 
A

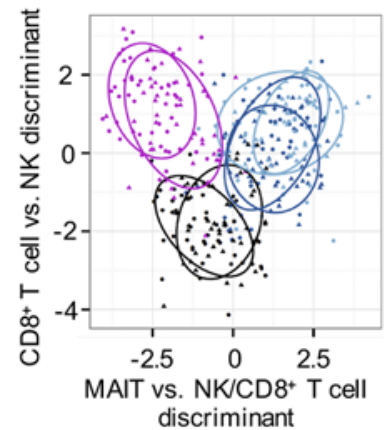

D
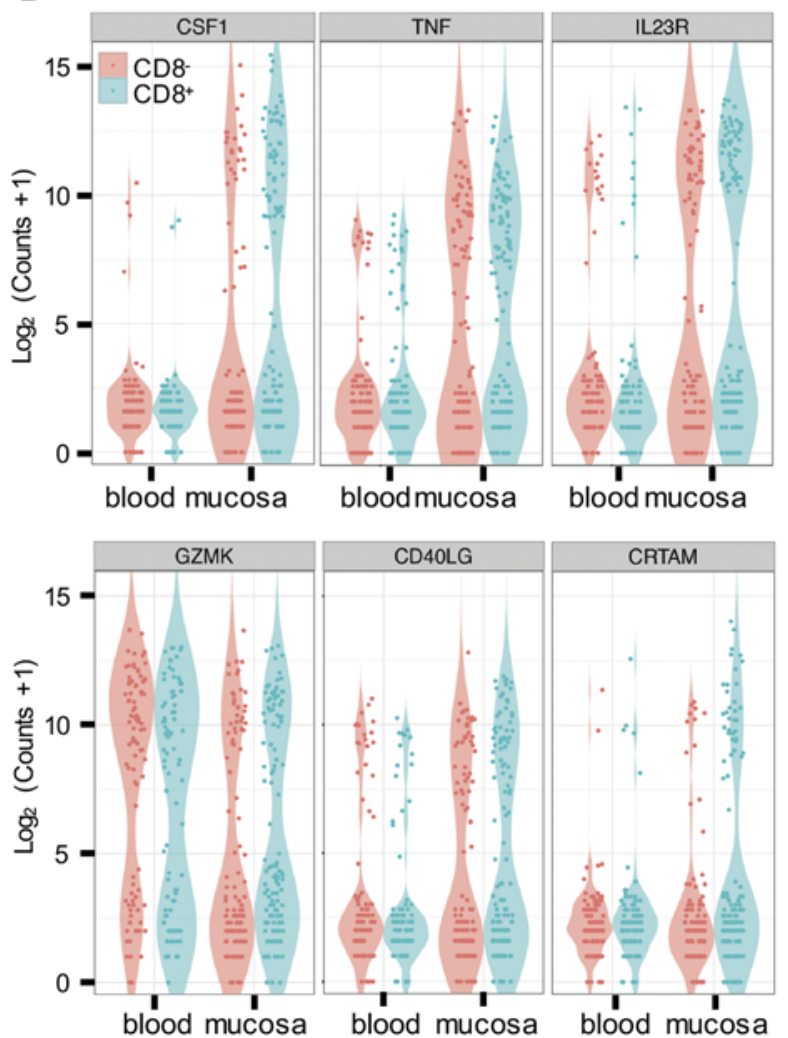

B
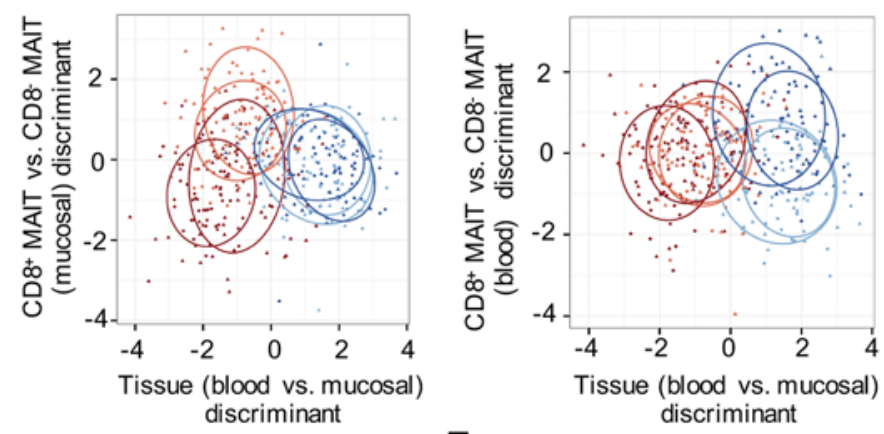

E

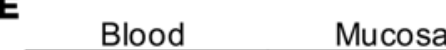

NK cell

$\mathrm{CD}^{+} \mathrm{T}$ cell

CD8+ blood MAIT

CD8- blood MAIT

CD8 ${ }^{+}$mucosal MAIT

CD8- mucosal MAIT

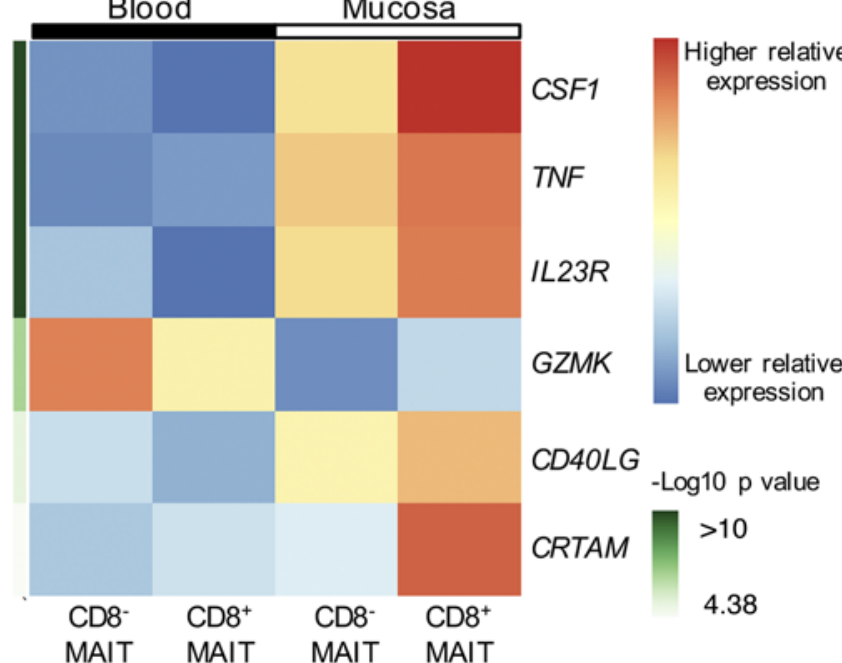

Higher relative

wer relative

CD4OLG Log10 p value

4.38

Figure 6. Mucosal MAIT cells are poised to respond more rapidly based on single cell gene expression analysis of MAIT cells isolated from blood and mucosal tissue. (A) Linear discriminant analysis of selected activation and inflammation genes expressed in CD8+ MAIT cells (dark blue), CD8- MAIT cells (light blue), CD8 ${ }^{+}$T cells (black), and NK cells (purple) isolated from the blood $(n=2)$. (B and C) CD8 ${ }^{+}$and CD8- MAIT cells from blood are compared with CD8 ${ }^{+}$(dark orange) and CD8- (light orange) MAIT cells isolated from rectal mucosal tissue. A confidence ellipse shows the expression profile of each donor $(n=2)$. (D) Violin plots of the top 6 genes that are differentially expressed (defined by Bonferroni significance) in CD8 ${ }^{+}$and CD8- MAIT cells from blood and mucosa show gene expression on a single cell level. Each single CD8+ (blue) and CD8- (red) MAIT cell in the blood and tissue is represented as a single dot ( $n=2$ donors). (E) Relative gene expression levels of the top 6 genes that are differentially expressed in CD8 ${ }^{+}$and CD8- MAIT cells from blood (columns 1 and 2) and mucosa (columns 3 and 4) are indicated in red (high) and and blue (low). Significant $P$ values comparing CD $8^{+}$and CD8- MAIT cells in blood and CD8 $8^{+}$and CD8- MAIT cells mucosal tissue are shown as $-\log _{10}$ values ranging from greater than 10 (dark green, top genes) to 4.38 (light green, bottom genes). See Methods for full statistical methods.

MAIT cells from blood and mucosal tissue (Figure 6, B and C, $x$-axis discriminant). We next interrogated which genes drive this tissue-based difference. Interestingly, we found that expression of genes associated with activating/proinflammatory functions (TNF, IL23R, CSF1, CD40L) was increased in mucosal MAIT cell populations, indicating that mucosal MAIT cells seem poised to respond more quickly compared with their counterparts in blood due to the increase in mRNA transcript (Figure 6, D and E). The increase in TNF gene expression in the mucosal MAIT cell populations does not result in the display of actual effector function directly ex vivo (Figure 7A) and thus may reflect the potential to respond rapidly upon activation, 
A

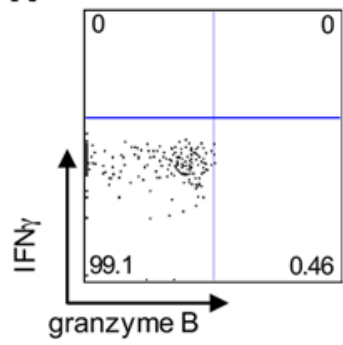

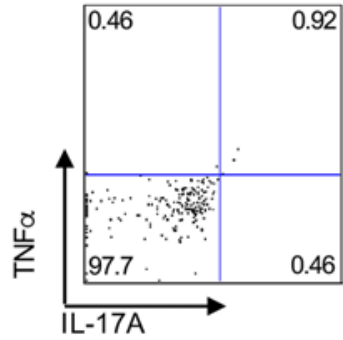

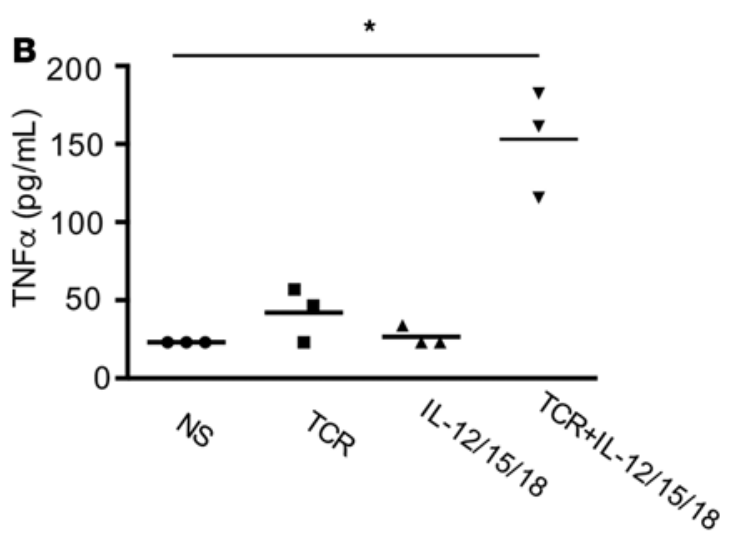

Figure 7. MAIT cells from healthy mucosal tissue do not secrete inflammatory cytokines ex vivo. (A) Representative FACS plot from one donor of cytokines produced directly ex vivo by CD8 ${ }^{+}$MAIT cells isolated from rectal mucosal biopsies. (B) Three thousand CD8 ${ }^{+}$CD161 ${ }^{\text {hiCCR6 }}$ hi cells were sorted from peripheral blood mononuclear cells (PBMC) and rested (NS, circles) and stimulated with anti-CD3/CD28 beads (TCR, squares), $100 \mathrm{ng} / \mathrm{ml}$ of IL-12/15/18 (triangles), or a combination of anti-CD3/28 beads plus IL-12/15/18 (TCR+IL-12/15/18, upside-down triangles) for 24 hours followed by analysis of the culture supernatants by Luminex $(n=3)$ for TNF- $\alpha$ secretion. TNF- $\alpha$ secretion that was below the limit of detection (LOD, $23 \mathrm{pg} / \mathrm{ml})$ is plotted at the LOD. ${ }^{*} P \leq 0.05$. $P$ values were determined using Mann-Whitney 1-tailed $U$ test (B).

as has been shown for other cytokines $(37,38)$. These data show that MAIT cells have an increased effector potential in healthy mucosal tissue and further underline the necessity for stringent regulation of their effector function. To further support the notion of stringent activation requirements, we sorted MAIT cells from PBMC and asked how to most effectively elicit a TNF- $\alpha$ response. Sorted MAIT cells were stimulated with IL-12/15/18 and/or via their TCR (Figure 7B). Robust TNF- $\alpha$ production occurred only when TCR signals synergized with inflammatory signals (Figure 7B), further underlining the need for both signals for inducing comprehensive MAIT cell effector function.

\section{Discussion}

Conventional $\mathrm{CD} 8^{+} \mathrm{T}_{\mathrm{mem}}$ and MAIT cells are important components of the immune system and have multiple overlapping characteristics. Both populations are fairly abundant in the blood, liver, and mucosal tissues, where they exert their effector function upon activation - specifically secretion of TNF- $\alpha$, IFN- $\gamma$, and granzyme B. MAIT cells express effector molecules in response to inflammatory signals and, as proposed by recent reports, also following TCR stimulation $(1,22,24)$. This would suggest that these 2 populations would respond in a very similar fashion and have overlapping function in response to inflammation and when encountering their specific antigen. However, we hypothesized that at least the sensitivity to TCRmediated signals for primary human MAIT cell and conventional $\mathrm{CD} 8^{+} \mathrm{T}_{\text {mem }}$ activation is different due to the nature of the antigen they recognize. Conventional CD8 T cells have a highly diverse TCR repertoire and recognize their cognate antigen (peptides with a typical length of 8-10 amino acids) in the context of MHC class I with great specificity. MAIT cells use an invariant TCR $\alpha$ chain and a limited repertoire of TCR $\beta$ chains to recognize a specific class of antigen, bacterial metabolites, and potentially metabolites of other origin, as well. Since many metabolic pathways are essential and conserved across bacterial species, MAIT cells cannot use their TCR to distinguish between metabolites from commensal versus pathogenic bacterial origin. Importantly, the current MAIT cell activation model suggests that a TCR signal is sufficient for inducing MAIT cell effector function. However, this model cannot explain how MAIT cells avoid responses against commensal-derived antigen or how MAIT cells could selectively respond to metabolites derived from commensal versus pathogenic bacteria.

To address these questions, we first wanted to clearly define which signals are sufficient to directly induce MAIT cell effector function ex vivo. We show here that an anti-CD3/CD28 signal is not sufficient to induce sustained robust effector function in FACS-purified MAIT cell populations (Figure 1B), while the same signal was sufficient to induce sustained effector function in $C D 8^{+} \mathrm{T}_{\text {mem }}$ (Figure 1C). Importantly, stimulating both populations with anti-CD3/CD28 beads has 2 important advantages: it allows us to directly compare the responses of both populations and it avoids the pitfall of using antigen, specifically metabolites, with still undefined TCR affinity. It is also noteworthy that a TCR signal is sufficient for MAIT 
cells to elicit a brief TNF- $\alpha$ and IFN- $\gamma$ pulse 6 hours after TCR stimulation. This is akin to what has been described in naive $\mathrm{CD} 8^{+} \mathrm{T}$ cells, which also briefly make IFN- $\gamma$ early after activation and prior to their first cell division (39). We could not detect significant production of IFN- $\gamma$ in MAIT cells by intracellular cytokine staining at later time points (Figure 1B) or in MAIT cell culture supernatant by Luminex analysis (Figure 2A) 24 hours after stimulation, further arguing that the production is very brief and limited. A previous study demonstrated that this brief IFN- $\gamma$ pulse does not result in antiviral immunity, and thus, the purpose is still unclear (39). An IFN- $\gamma$ response following TCR stimulation was reported in MAIT cell clones and lines $(28,40)$, which are useful tools to identify antigens but not a suitable substitute for studying ex vivo activation requirements of primary human $\mathrm{T}$ cells.

Although a TCR signal is not sufficient to induce robust effector function in MAIT cells, primary human MAIT cells still respond to a TCR + costimulatory signal. However, instead of eliciting prolonged, robust effector function, a TCR + costimulatory signal elicits secretion of the chemokines CCL3 and CCL4 (Figure 2B). Similarly, conventional memory CD8 ${ }^{+} \mathrm{T}$ cells secrete CCL3 and CCL4 in response to TCR stimulation (Figure 2B). The functions of CCL3 and CCL4 include recruitment of monocytes (41), which may serve as a surveillance population. Thus, our data demonstrate that MAIT cells do not secrete significant levels of effector molecules following TCR stimulation but are not generally hyporesponsive to TCR signaling based on their ability to express CCL3 and CCL4 (Figure 2B). This phenotype resembles the altered TCR responsiveness of other innate-like T cells previously demonstrated in a mouse model system (42) and CD161 1 hi CD8 $\alpha^{+} \mathrm{T}$ cells (43). More work is needed to determine the downstream signaling cascade when MAIT cells are stimulated via their TCR. Similarly, the indirect signals that lead to activation of MAIT cells when bulk PBMCs are activated with anti-CD3/CD28 stimuli (Figure 3, A and B) are still undefined. It is important to consider that additional mechanisms may be in place to further control MAIT cell function: for example, MAIT cell exposure to antigen could be further controlled via regulation of MR1 expression, which does not appear to be constitutively expressed on the cell surface $(44,45)$. In summary, our data argue for a fundamentally different role of a TCR signal for MAIT cells versus CD8 ${ }^{+}$ $\mathrm{T}_{\mathrm{mem}}$ to elicit effector function.

We and others have previously shown that inflammatory cues (IL-12/15/18) are sufficient to directly activate MAIT cells $(22,24)$. We demonstrate here that the MAIT cell population responds more vigorously compared with the $\mathrm{CD}^{+} \mathrm{T}_{\text {mem }}$ population (Figure 1, B and C, and Figure 3, A and B), suggesting that MAIT cells also play an important role in inflammatory processes in the absence of their cognate antigen. Following cytokine-induced activation, conventional $\mathrm{CD}^{+} \mathrm{T}_{\text {mem }}$ can kill target cells in an NKG2D-dependent and TCR-independent manner $(7,11,14,18,46)$. Future studies will need to examine if MAIT cells can similarly exert direct cytotoxic effector function without cognate antigen recognition by the TCR. To more stringently test the ability of MAIT cells to respond in an inflammation-driven activation scenario under physiologically relevant conditions, we examined the ability of MAIT cells to respond to monocytes that were activated with ssRNA, a TLR8 agonist. We specifically asked whether TLR-stimulated monocytes could activate MAIT cells in a cell contact-dependent and-independent manner by culturing MAIT cells with monocytes or monocyte supernatant as outlined in Figure 4. MAIT cells acquired effector function following incubation with supernatant alone, demonstrating that direct monocyte-to-MAIT cell contact is not necessary for activation (Figure 4, A and B).

Together, the experiments described so far allowed us to define the respective contributions of inflammatory versus TCR signals to inducing MAIT cell effector function. To determine how TCR and cytokine signals integrate to activate MAIT cells, we either delivered a TCR signal, a cytokine signal (supernatant from monocytes that were stimulated with ssRNA or LPS), or a combination of TCR + cytokine signals (Figure 5, A and B). Importantly, the latter combination of signals led to a synergistic increase in effector function, regardless of the nature of the TLR agonist. Only TLR8- but not TLR4-stimulated monocytes secreted detectable amounts of IL-12p70 (Figure 4C and Figure 5C). However, both supernatants were sufficient to elicit MAIT cell effector function, which suggests that inflammatory cues other than IL-12 and IL-18 are sufficient to synergize with the TCR signal to induce effector function. Future studies will need to determine the nature of these cytokines.

Finally, we sought to compare MAIT cells from blood and mucosal tissue to determine if stringent control of effector functions is necessary for MAIT cells located in tissues. We considered the possibility that MAIT cells in the tissues may have inherently lower functional potential to prevent unwanted responses in healthy tissues. Importantly, our single cell gene expression analysis showed enhanced immediate effec- 
tor potential in a large portion of the mucosal MAIT cell population (Figure 6, D and E). The single cell analysis shows a biphasic distribution within the mucosal MAIT cell population for most genes (Figure $6 \mathrm{D}$, violin plots). This distribution may be due to the specific location of MAIT cells in the tissue or due to the amount of time the cells have been in the tissue, and there could also be a stochastic component. Future studies will need to address the underlying mechanisms. Interestingly, granzyme $\mathrm{K}(G Z M K)$ was an exception in that its expression was decreased in mucosal tissue-derived MAIT cells (Figure 6, D and E). Granzyme K can enhance LPS-induced cytokine secretion from human monocytes (47). The decrease in GZMK expression we observed could serve to prevent unwanted cytokine responses against commensal-derived LPS, thus avoiding eliciting an inflammatory response and induction of MAIT cell effector function. Finally, TNF- $\alpha$ and other cytokines are not expressed on a protein level by MAIT cells in healthy mucosal tissue directly ex vivo (Figure 7A). This demonstrates that the increase in transcripts of effector molecules in mucosal MAIT cells does not indicate an ongoing effector response but instead suggests that mucosal MAIT cells are poised to respond quickly.

In summary, our data provide, to our knowledge, a novel model of human MAIT cell activation requirements. Since inflammatory signals are necessary for acquisition of robust MAIT cell effector function and synergize with TCR signals, we propose that this previously unappreciated requirement for acquiring MAIT cell effector function provides an explanation for the coexistence of MAIT cells and commensals in mucosal tissues without eliciting pathology (TCR signal without inflammation, ref. 48), while maintaining responsiveness to pathogens (TCR signal plus inflammation). Future studies will address the consequences of (wanted and unwanted) MAIT cell activation in mucosal tissue and their role in inflammatory disorders of the mucosal tissue (49), including bacterial translocation following HIV/SIV infection $(32,50)$ as well as intestinal inflammation following BM transplantation (30). Importantly, MAIT cells express the drug efflux reporter ABCB1, which allows them to preferentially survive cytotoxic chemotherapy $(23,51)$. In the latter context, it will be of particular interest to investigate the effect of MAIT cell activation on the diversity of the microbiome, which has been implicated in directly affecting patient outcome (52-54). Importantly, our study outlines the mechanisms for inducing MAIT cell effector function and how they could be targeted for improving prevention and treatment strategies.

\section{Methods}

Flow cytometry. For the phenotypic identification or functional assays, bulk PBMC or sorted MAIT cells were stained with Aqua Live/Dead Fixable Dead Cell Stain (Invitrogen) and a combination of the following antibodies (from BD Biosciences except as noted): CD3 Pacific Blue (clone OKT3, BioLegend), CD8 PerCPCy5.5 (clone SK1), CD4 ECD (clone SFCI12T4D11, Beckman Coulter), CD161 PECy5 (clone DX12), Va7.2 PE (clone 3C10, BioLegend), CD56 PECy7 (clone NCAM16.2), CD16 APCCy7 (clone 3G8), granzyme B (clone GB11), IFN- $\gamma$ (clone 4S.b3, eBioscience), TNF- $\alpha$ (clone MAb11), IL-17A (clone SHLR17, eBioscience), CD45RO (clone UCHL1), CCR6 (clone R6H1, eBioscience), and CD62L (clone DREG-56). MAIT cells that were enriched for $\mathrm{CD} 8^{+} \mathrm{CD} 161^{\mathrm{hi}}$-expressing cells were sorted as live $\mathrm{CD} 4^{-} \mathrm{CD} 8^{+} \mathrm{CD} 56^{+} \mathrm{CD} 16^{-} \mathrm{V} \alpha 24^{-} \mathrm{CD} 161^{\mathrm{hi}}$ cells. MAIT cells that were enriched for high purity based on CCR6 expression were sorted as live $\mathrm{CD} 4^{-} \mathrm{CD} 8^{+} \mathrm{CD} 56^{+} \mathrm{CD} 16^{-} \mathrm{CD} 161^{\mathrm{hi}} \mathrm{CCR} 6^{\mathrm{hi}} \mathrm{CD} 62 \mathrm{~L}^{\text {lo }}$ cells. MAIT cells that were enriched for purity based on Va7.2 expression were sorted as live $\mathrm{CD} 4{ }^{-} \mathrm{CD} 8^{+} \mathrm{CD} 56^{+} \mathrm{CD} 16^{-} \mathrm{CD} 161^{\mathrm{hi}} \mathrm{V} \alpha 7.2^{+}$cells. Sorted purities were typically greater than $90 \%-95 \%$ and performed on a FACS Aria II (BD Biosciences). For the phenotypic identification of conventional $\mathrm{CD} 8^{+} \mathrm{T}\left(\mathrm{CD} 8^{+} \mathrm{T}_{\mathrm{mem}}\right)$ cells, bulk PBMC or sorted $\mathrm{CD} 8^{+} \mathrm{T}_{\text {mem }}$ cells were stained with Aqua Live/Dead Fixable Dead Cell Stain and a combination of antibodies. CD8 $8^{+} \mathrm{T}_{\text {mem }}$ cells that were enriched for purity were sorted as live $\mathrm{CD} 4^{-} \mathrm{CD} 8^{+} \mathrm{CD} 45 \mathrm{RO}^{+} \mathrm{V} \alpha 7 \cdot 2^{-}$cells.

Activation of cells. Cells were left untreated or were treated with one dose of IL-12 (eBioscience), IL-15 (eBioscience), and IL-18 (MBL) at $100 \mathrm{ng} / \mathrm{ml}$ or a combination of the cytokines and anti-CD3/CD28coupled beads per manufacturers instructions for whole PBMC and increased to a 10:1 bead/cell ratio to ensure activation of sorted MAIT or $\mathrm{CD}^{+} \mathrm{T}_{\text {mem }}$ cultures (Invitrogen) and cultured for 6, 12, or 24 hours. Whole PBMC were stimulated at a concentration of $1 \times 10^{6}$ cells per well, and sorted cells were stimulated at a concentration of 5,000 cells per well. Golgi plug (BD Biosciences) was added 4 hours before supernatant harvest and intracellular staining at each time point. Supernatant was collected from cultures where indicated 24 hours after stimulation. Cells were analyzed by FACS and Luminex according to the manufacturer's instructions (Luminex Corp.).

Monocyte isolation and stimulation. Cryopreserved PBMC were thawed and immediately prepared for 
$\mathrm{CD} 14^{+}$monocyte isolation using a CD14${ }^{+}$isolation kit (Miltenyi Biotec). CD $14^{+}$monocytes were left untreated or treated with either LPS at $1 \mathrm{ng} / \mathrm{ml}$ or ssRNA at $1 \mu \mathrm{g} / \mathrm{ml}$ (Invivogen) for 24 hours in a $37^{\circ} \mathrm{C}$ incubator. Cytokine concentrations within supernatants were assessed by Luminex. Following culture, MAIT cells were analyzed for activation by flow cytometry.

Culture of MAIT cells with TLR-activated monocytes. After 24 hours of monocyte activation, MAIT cells were sorted from a separate vial of cryopreserved PBMC from the same donor and cultured with monocytes at a 1:10 ratio or treated with 100-150 $\mu$ of supernatant from monocyte cultures for an additional 24 hours. They were then stained for MAIT cell markers and cytokine expression.

Isolation of cells from mucosal tissue. Five $3-\mathrm{mm}^{2}$ punch rectal biopsy specimens, obtained $10 \mathrm{~cm}$ proximal to the anal verge, were collected during the same visit as blood was collected, transported on wet ice, and placed immediately into RPMI 1640 supplemented with penicillin $(100 \mathrm{U} / \mathrm{ml})$, streptomycin sulfate (100 $\mu \mathrm{g} / \mathrm{ml}$ ), and Fungizone $(2.5 \mu \mathrm{g} / \mathrm{ml}$ ) (all from Gibco BRL). They were repeatedly washed, and mucosal mononuclear cells (MMC) were isolated by 2 rounds of digestion with collagenase II (Sigma-Aldrich). Cells were immediately stained for single cell sorting described below.

Ex vivo cytokine staining of blood and mucosal MAIT cells. Golgi plug was added for 4 hours to MMC before staining MAIT cells for IFN- $\gamma$, granzyme B, TNF- $\alpha$, and IL-17A expression. MAIT cells are identified by gating on live, $\mathrm{CD} 45^{+} \mathrm{CD} 3^{+} \mathrm{CD} 8^{+} \mathrm{CD} 161^{\text {hiv }} \alpha 7.2^{+}$cells.

Single cell gene expression experiments. For Nanostring experiments, CD8 ${ }^{+}$MAIT cells were sorted as live $\mathrm{CD}^{+}{ }^{+} \mathrm{CD} 8^{+} \mathrm{CD} 4^{-} \mathrm{CD} 161^{\mathrm{hi}} \mathrm{V} \alpha 7.2^{+}$cells, CD8 ${ }^{-}$MAIT cells were sorted as live CD3 ${ }^{+} \mathrm{CD} 8^{-} \mathrm{CD} 4^{-} \mathrm{CD} 161^{\mathrm{hi}}$ $\mathrm{V} \alpha 7.2^{+}$cells, $\mathrm{CD}^{+}$conventional $\mathrm{T}$ cells were sorted as live $\mathrm{CD} 3^{+} \mathrm{CD} 8^{+} \mathrm{CD} 4{ }^{-} \mathrm{CD} 161^{-} \mathrm{V} \alpha 7.2^{-}$cells, and $\mathrm{NK}$ cells were sorted as live $\mathrm{CD}^{-} \mathrm{CD} 56^{+} \mathrm{CD} 16^{+}$cells. Single cells were sorted into 96-well polypropylene PCR plates (Eppendorf) containing lysis buffer (NanoString Technologies) and immediately frozen. After lysis, RNA was converted to cDNA with SuperScript VILO (Invitrogen). Primers for 145 genes were pooled, and cDNA was enriched in a multiplexed amplification (MTE) reaction according to the nCounter Single Cell Expression protocol (NanoString Technologies). The MTE samples were hybridized overnight at $65^{\circ} \mathrm{C}$ with an nCounter CodeSet containing probes for all enriched targets (IAM, unrelated genes, and controls) and internal controls as recommended by the manufacturer. Gene expression was analyzed using the nCounter system (NanoString Technologies).

Statistical and computational methods for MAIT NanoString experiment. A total of 756 wells containing single cells were sampled. Single cell gene expression data were quality controlled and preprocessed as previously described $(55,56)$. After filtering, 701 cells remained. Forty percent of filtered cells corresponded to wells without detectable expression. We did not consider any explicit normalization, as single cells were sampled, providing natural, atomic units of input RNA normalization.

Multivariate classification and dimension reduction. We considered to what degree gene expression patterns could discriminate cells into their (i) cell subset within blood and tissue lymphocytes and (ii) tissue type within MAITs. To do this, we used Fisher's linear discriminant analysis (LDA). LDA performed on cells belonging to $K$ distinct phenotypic groups results in the gene expression vector profile of each cell being reduced to a set of $K-1$ canonical coordinates via a linear transformation of the gene expression vector. This is akin to doing principal components analysis in a manner that maximizes the spread between the $K$ groups, i.e., between cell subsets and tissue types.

We considered LDA of lymphocytes in blood (Figure 6A) and within MAITs across tissue type (Figure $6, \mathrm{~B}$ and $\mathrm{C}$ ) and plotted the location of each blood and mucosa sample according to the LDA ordination. Out of the 3 canonical coordinates to separate the 4 cell types collected from blood, the coordinates that discriminate MAITs and NK from all other cell types are shown in Figure 6A. In Figure 6, B and C, the coordinate that discriminates between tissues in MAITs and the 2 coordinates that discriminate $\mathrm{CD}^{+/-}$in each tissue type are shown. Ellipses are $75 \%$ confidence regions of the location (centroid) of the points for each donor and cell type assuming bivariate normality.

Differential Gene Expression. Using the Hurdle linear model $(55,56)$, developed to accommodate bimodality in single cell gene expression, we tested for changes between tissues by $\mathrm{CD}^{+/-}$status. A total of 28 genes had any difference between subsets ( 6 degree of freedom $\chi^{2}$ test, $5 \%$ Bonferroni significant across IAM genes). The differences were further decomposed into an additive effect due to tissue differences, an additive effect due to $\mathrm{CD} 8^{+/-}$status, and an interaction between tissue and $\mathrm{CD} 8^{+/-}$status. Genes with consistent tissue differences were found by screening for genes with stable $\mathrm{CD}^{+/-}$patterns and no evidence of an interaction between CD8 status and tissue (FDR q-values $>10 \%$ ). The genes with the 6 most significant 
tissue differences (all Bonferroni significant < 1\%) are shown in Figure 6, D and E. We calculate the signed signal/noise ratio by taking the average log expression for each of the 4 subtypes, centering 4 subtype averages at about 0 , and dividing by the pooled standard deviation of single cell expression. Genes with larger differences between subgroups with respect to the residual single cell variability in the gene have more extreme heat map values.

Statistics. Nonparametric tests were performed using Mann-Whitney or 2-way ANOVA tests as appropriate and corrected for multiple comparisons using Dunnett's test. $P$ values greater than 0.05 were considered not significant (ns), and values denoted with $\left(^{*}\right)$ symbols reflect significance levels as follows: $P \leq$ $0.05\left(^{*}\right), P \leq 0.01\left(^{* *}\right), P \leq 0.001\left(^{* * *}\right)$, and $P \leq 0.0001\left(^{* * *}\right)$. Stimulated samples were compared with the negative control using a 1-tailed test and to each other using a 2-tailed test. Analyses were performed using GraphPad software (GraphPad Prism) version 6.0e.

Study approval. Twelve healthy, HIV-uninfected adults (7 male, 5 female; age range of 31-60 years) were recruited at the Seattle HIV Vaccine Trials Unit (Seattle, Washington, USA) as part of the study "Establishing Immunologic Assays for Determining HIV-1 Prevention and Control", also referred to as Seattle Assay Control or SAC. All participants signed informed consent, and the following institutional human subjects review committee approved the protocol prior to study initiation: Fred Hutchinson Cancer Research Center IRB (Seattle, Washington, USA).

\section{Author Contributions}

CKS and MP conceived and designed experiments. CKS, AM, and MP wrote the paper. CKS, MP, HWM, BJS, and JPM performed experiments and analyzed data. AM, GF, and RG analyzed single cell gene expression data, contributed to data interpretation, and provided manuscript feedback. GD, JLC, and MJM contributed advice, reagents, and materials; enrolled human subjects; and provided manuscript feedback. JLC collected blood and performed tissue biopsies.

\section{Acknowledgments}

This work was supported by NIH grants DP2 DE023321 (to M. Prlic), R01 EB008400 (to R. Gottardo), and T32 AI007509 (to C.K. Slichter). We thank Lucas Dennis and Mike Krouse for assistance with the NanoString single cell gene expression experiments; all study volunteers and the Seattle HIV Vaccine Trials Unit for providing samples; Noel Williams and the Seattle VTU for recruiting and scheduling participants; Dietmar Zehn, Steve Jameson, Stan Riddell, and Cameron Turtle for helpful discussions; and Stephen Voght for critical reading and editing of the manuscript. We thank the James B. Pendleton Charitable Trust for their generous equipment donation.

Address correspondence to: Martin Prlic, Fred Hutchinson Cancer Research Center, Vaccine and Infectious Disease Division, 1100 Fairview Ave N E5-110, PO Box 19024, Seattle, WA 98109-1024. Phone: 206.667.2216; E-mail: mprlic@fhcrc.org.

1. Chandra S, Kronenberg M. Activation and function of iNKT and MAIT cells. Adv Immunol. 2015;127:145-201.

2. Tough DF, Borrow P, Sprent J. Induction of bystander T cell proliferation by viruses and type I interferon in vivo. Science. 1996;272(5270):1947-1950.

3. Carding SR, Allan W, McMickle A, Doherty PC. Activation of cytokine genes in T cells during primary and secondary murine influenza pneumonia. J Exp Med. 1993;177(2):475-482.

4. Berg RE, Cordes CJ, Forman J. Contribution of CD8 ${ }^{+} \mathrm{T}$ cells to innate immunity: IFN-gamma secretion induced by IL-12 and IL-18. Eur J Immunol. 2002;32(10):2807-2816.

5. Berg RE, Crossley E, Murray S, Forman J. Memory CD8 ${ }^{+}$T cells provide innate immune protection against Listeria monocytogenes in the absence of cognate antigen. J Exp Med. 2003;198(10):1583-1593.

6. Soudja SM, Ruiz AL, Marie JC, Lauvau G. Inflammatory monocytes activate memory CD8(+) T and innate NK lymphocytes independent of cognate antigen during microbial pathogen invasion. Immunity. 2012;37(3):549-562.

7. Chu T, et al. Bystander-activated memory CD8 T cells control early pathogen load in an innate-like, NKG2D-dependent manner. Cell Rep. 2013;3(3):701-708.

8. Odumade OA, Knight JA, Schmeling DO, Masopust D, Balfour HH Jr., Hogquist KA. Primary Epstein-Barr virus infection does not erode preexisting CD8(+) T cell memory in humans. J Exp Med. 2012;209(3):471-478.

9. Jiang W, et al. Cycling memory $\mathrm{CD}^{+} \mathrm{T}$ cells in HIV disease have a diverse $\mathrm{T}$ cell receptor repertoire and a phenotype consistent with bystander activation. $J$ Virol. 2014;88(10):5369-5380.

10. Shive CL, et al. Inflammatory cytokines drive CD4+ T-cell cycling and impaired responsiveness to interleukin 7: implications for immune failure in HIV disease. J Infect Dis. 2014;210(4):619-629. 
11. Sckisel GD, et al. Influenza infection results in local expansion of memory CD8(+) T cells with antigen non-specific phenotype and function. Clin Exp Immunol. 2014;175(1):79-91.

12. Bou Ghanem EN, D'Orazio SE. Human $\mathrm{CD}^{+} \mathrm{T}$ cells display a differential ability to undergo cytokine-driven bystander activation. Cell Immunol. 2011;272(1):79-86.

13. Doisne JM, et al. CD8 ${ }^{+} \mathrm{T}$ cells specific for EBV, cytomegalovirus, and influenza virus are activated during primary HIV infection. J Immunol. 2004;173(4):2410-2418.

14. Tietze JK, et al. Delineation of antigen-specific and antigen-nonspecific CD8(+) memory T-cell responses after cytokine-based cancer immunotherapy. Blood. 2012;119(13):3073-3083.

15. Kohlmeier JE, Cookenham T, Roberts AD, Miller SC, Woodland DL. Type I interferons regulate cytolytic activity of memory CD8(+) T cells in the lung airways during respiratory virus challenge. Immunity. 2010;33(1):96-105.

16. Crosby EJ, Clark M, Novais FO, Wherry EJ, Scott P. Lymphocytic choriomeningitis virus expands a population of NKG$2 \mathrm{D}^{+} \mathrm{CD}^{+} \mathrm{T}$ cells that exacerbates disease in mice coinfected with Leishmania major. J Immunol. 2015;195(7):3301-3310.

17. Crosby EJ, Goldschmidt MH, Wherry EJ, Scott P. Engagement of NKG2D on bystander memory CD8 T cells promotes increased immunopathology following Leishmania major infection. PLoS Pathog. 2014;10(2):e1003970.

18. Meresse B, et al. Coordinated induction by IL15 of a TCR-independent NKG2D signaling pathway converts CTL into lymphokine-activated killer cells in celiac disease. Immunity. 2004;21(3):357-366.

19. Holzapfel KL, Tyznik AJ, Kronenberg M, Hogquist KA. Antigen-dependent versus -independent activation of invariant NKT cells during infection. J Immunol. 2014;192(12):5490-5498.

20. Tyznik AJ, Tupin E, Nagarajan NA, Her MJ, Benedict CA, Kronenberg M. Cutting edge: the mechanism of invariant NKT cell responses to viral danger signals. J Immunol. 2008;181(7):4452-4456.

21. Tyznik AJ, Verma S, Wang Q, Kronenberg M, Benedict CA. Distinct requirements for activation of NKT and NK cells during viral infection. J Immunol. 2014;192(8):3676-3685.

22. Ussher JE, et al. CD161(++) CD8(+) T cells, including the MAIT cell subset, are specifically activated by IL-12+IL-18 in a TCR-independent manner. Eur J Immunol. 2014;44(1):195-203.

23. Dusseaux M, et al. Human MAIT cells are xenobiotic-resistant, tissue-targeted, CD161hi IL-17-secreting T cells. Blood. 2011;117(4):1250-1259.

24. Jo J, et al. Toll-like receptor 8 agonist and bacteria trigger potent activation of innate immune cells in human liver. PLoS Pathog. 2014;10(6):e1004210.

25. Finak G, et al. MAST: a flexible statistical framework for assessing transcriptional changes and characterizing heterogeneity in single-cell RNA sequencing data. Genome Biol. 2015;16(1):278.

26. Gapin L. Check MAIT. J Immunol. 2014;192(10):4475-4480.

27. Magalhaes I, Kiaf B, Lehuen A. iNKT and MAIT cell alterations in diabetes. Front Immunol. 2015;6:341.

28. Kjer-Nielsen L, et al. MR1 presents microbial vitamin B metabolites to MAIT cells. Nature. 2012;491(7426):717-723.

29. Salerno-Goncalves R, Rezwan T, Sztein MB. B cells modulate mucosal associated invariant T cell immune responses. Front Immunol. 2014;4:511.

30. Hill GR. Inflammation and bone marrow transplantation. Biol Blood Marrow Transplant. 2009;15(1 Suppl):139-141.

31. Klase Z, et al. Dysbiotic bacteria translocate in progressive SIV infection. Mucosal Immunol. 2015;8(5):1009-20.

32. Klatt NR, Funderburg NT, Brenchley JM. Microbial translocation, immune activation, and HIV disease. Trends Microbiol. 2013;21(1):6-13.

33. Cosgrove C, et al. Early and nonreversible decrease of CD161++/MAIT cells in HIV infection. Blood. 2013;121(6):951-961.

34. Leeansyah E, et al. Activation, exhaustion, and persistent decline of the antimicrobial MR1-restricted MAIT-cell population in chronic HIV-1 infection. Blood. 2013;121(7):1124-1135.

35. Jakubzick C, et al. Minimal differentiation of classical monocytes as they survey steady-state tissues and transport antigen to lymph nodes. Immunity. 2013;39(3):599-610.

36. Treiner E, et al. Selection of evolutionarily conserved mucosal-associated invariant T cells by MR1. Nature. 2003;422(6928):164-169.

37. Swanson BJ, Murakami M, Mitchell TC, Kappler J, Marrack P. RANTES production by memory phenotype T cells is controlled by a posttranscriptional, TCR-dependent process. Immunity. 2002;17(5):605-615.

38. Mohrs K, Wakil AE, Killeen N, Locksley RM, Mohrs M. A two-step process for cytokine production revealed by IL-4 dual-reporter mice. Immunity. 2005;23(4):419-429.

39. Hosking MP, Flynn CT, Whitton JL. Antigen-specific naive CD8 ${ }^{+}$T cells produce a single pulse of IFN-gamma in vivo within hours of infection, but without antiviral effect. J Immunol. 2014;193(4):1873-1885.

40. Gold MC, et al. Human mucosal associated invariant T cells detect bacterially infected cells. PLoS Biol. 2010;8(6):e1000407.

41. Maurer M, von Stebut E. Macrophage inflammatory protein-1. Int J Biochem Cell Biol. 2004;36(10):1882-1886.

42. Wencker $\mathrm{M}$, et al. Innate-like T cells straddle innate and adaptive immunity by altering antigen-receptor responsiveness. Nat Immunol. 2014;15(1):80-87.

43. Turtle CJ, et al. Innate signals overcome acquired TCR signaling pathway regulation and govern the fate of human CD161(hi) CD8alpha(+) semi-invariant T cells. Blood. 2011;118(10):2752-2762.

44. Hashimoto K, Hirai M, Kurosawa Y. A gene outside the human MHC related to classical HLA class I genes. Science. 1995;269(5224):693-695.

45. Chua WJ, et al. Endogenous MHC-related protein 1 is transiently expressed on the plasma membrane in a conformation that activates mucosal-associated invariant T cells. J Immunol. 2011;186(8):4744-4750.

46. Verneris MR, Karimi M, Baker J, Jayaswal A, Negrin RS. Role of NKG2D signaling in the cytotoxicity of activated and expanded CD8 ${ }^{+}$T cells. Blood. 2004;103(8):3065-3072.

47. Wensink AC, et al. Granzyme K synergistically potentiates LPS-induced cytokine responses in human monocytes. Proc Natl Acad Sci U S A. 2014;111(16):5974-5979.

48. Ramanan D, Tang MS, Bowcutt R, Loke P, Cadwell K. Bacterial sensor Nod2 prevents inflammation of the small intestine by restricting the expansion of the commensal Bacteroides vulgatus. Immunity. 2014;41(2):311-324. 
49. Belkaid Y, Hand TW. Role of the microbiota in immunity and inflammation. Cell. 2014;157(1):121-141.

50. Klatt NR, et al. Compromised gastrointestinal integrity in pigtail macaques is associated with increased microbial translocation, immune activation, and IL-17 production in the absence of SIV infection. Mucosal Immunol. 2010;3(4):387-398

51. Turtle CJ, Swanson HM, Fujii N, Estey EH, Riddell SR. A distinct subset of self-renewing human memory CD8 ${ }^{+}$T cells survives cytotoxic chemotherapy. Immunity. 2009;31(5):834-844.

52. Jenq RR, et al. Regulation of intestinal inflammation by microbiota following allogeneic bone marrow transplantation. $J$ Exp Med. 2012;209(5):903-911.

53. Taur Y, et al. The effects of intestinal tract bacterial diversity on mortality following allogeneic hematopoietic stem cell transplantation. Blood. 2014;124(7):1174-1182.

54. Docampo MD, Auletta JJ, Jenq RR. Emerging influence of the intestinal microbiota during allogeneic hematopoietic cell transplantation: Control the gut the body will follow. Biol Blood Marrow Transplant. 2015;21(8):1360-1366.

55. McDavid A, et al. Modeling bi-modality improves characterization of cell cycle on gene expression in single cells. PLoS Comput Biol. 2014;10(7):e1003696.

56. McDavid A, et al. Data exploration, quality control and testing in single-cell qPCR-based gene expression experiments Bioinformatics. 2013;29(4):461-467. 